\title{
Review of APOS Theory: A Framework for Research and Curriculum Development in Mathematics Education, Arnon et al. (2014). New York, NY, USA: Springer-Verlag New York. eBook ISBN: 978-1-4614-7966-6, Hardcover ISBN: 978-1-4614-7965-9
}

\author{
Matthew Inglis ${ }^{1}$
}

Published online: 16 October 2015

(C) Springer International Publishing Switzerland 2015

APOS theory holds an important place in the history of research on undergraduate mathematics education, especially in the US-based RUME community (e.g., Selden 2012). Developed in the 80s, the theory was elaborated in Dubinsky's (1991) chapter in the influential Advanced Mathematical Thinking text (Tall 1991), and has since been developed and applied to various domains across many journal articles and conference papers. APOS theorists were among the first to pay serious attention to how students come to understand mathematical concepts, and to think carefully about how this should inform pedagogic interventions. That there is now a blossoming field of research in undergraduate mathematics education is, to a large extent, due to the group of researchers who first worked on APOS theory. But the theory is perhaps not as dominant as it once was, and this book is clearly intended to persuade researchers to consider adopting an APOS-based approach. Indeed, in their preface, the authors state that their aim is to offer a detailed account of the theory, with the goal of contributing towards the education of graduate students and young mathematics education researchers.

The ambition of the book is substantial: the authors describe APOS theory as a Kuhnian paradigm, and set out to explain the paradigmatic questions, assumptions, and methods that within-paradigm researchers adopt. Kuhn (1962) suggested that paradigms, during normal science at least, are universally accepted within a given discipline. The existence of theoretical rivals to APOS in the mathematics education literature suggests that the authors' description might not be quite correct, and that

I am very grateful to Keith Weber for thoughtful comments on an earlier draft of this review. This work was supported by a Royal Society Worshipful Company of Actuaries Research Fellowship.

Matthew Inglis

m.j.inglis@lboro.ac.uk

1 Mathematics Education Centre, Loughborough University, Loughborough, UK 
APOS theory might be better characterised as a Lakatosian research programme. I will suggest that, as well as being a more accurate description of APOS theory's status, Lakatos's (1976) notion of a research programme provides a helpful structure through which we can evaluate its contribution.

In The Methodology of Scientific Research Programmes, Lakatos (1976) suggested that a research programme has several components. It has a 'hard core', the key assumptions and beliefs that define the programme. Alongside the hard core is the programme's 'heuristic', a set of problem-solving techniques that researchers use within the programme to make progress and solve problems. In addition to the hard core and heuristic, a research programme also has a 'protective belt' of auxiliary hypotheses, the goal of which is to protect the hard core from empirical anomalies. Whenever an anomaly appears-whenever an empirical finding causes difficulties for the current version of the theory-a 'rescue hypothesis' can be added to the protective belt. In other words, the protective belt, but not the hard core, can simply be modified to absorb the anomaly.

Lakatos distinguished between two types of research programme. A progressing programme is one which successfully absorbs anomalies and turns them to its advantage by regularly generating surprising new predictions and results. Because of its success, we should have relatively high levels of confidence in the validity of a progressing programme's hard core. In contrast, a degenerating programme rarely makes novel discoveries or predictions, and dedicates its protective belt to the explanation and accommodation of anomalies. Lakatos suggested that scientific revolutions occur when researchers give up trying to accommodate anomalies into a degenerating research programme, and replace it by an alternative research programme that is progressing.

So which is APOS theory? A progressing research programme or a degenerating one? I thought about this question a lot while reading the book. My conclusion is that it's very hard to tell. Very few, if any, Lakatosian anomalies are discussed by the authors. This could be because there aren't any: perhaps the research literature contains no results that cause difficulties for the APOS hard core. But I do not think that this is the case, and later in the review give an explicit example of an anomaly that needs to be accommodated. An alternative possibility, which to me seems more likely, is that the research programme has not paid sufficient attention to the wider literature and possible anomalies it contains.

Before discussing these issues further, I briefly give an account of APOS's hard core and heuristic. The fundamental assumption of APOS theory is that there are three basic types of mathematical knowledge: actions, processes and objects. Actions are physical or mental manipulations of objects that result in other objects. For example, if a child adds 4 to 3 to get 7 by counting on, they have performed an action. If a learner is able to reflect upon an action in a way that allows them to think about it without needing to explicitly perform it, they have interiorized the action into a process. So, a child may be able to think about the process of adding 4 without needing to explicitly do it. Once they have interiorized the action, they will be able to reverse it, and to skip steps. For instance if the child is able to reverse 'add 4' by subtracting 4, this would be evidence that they had interiorized the 'add 4' action. Sometimes processes can act as objects for new actions. In this case the learner is said to have 
encapsulated the process into an object. Schemas are coherent collections of the three more basic types: actions, processes and objects that are related in some way. This repeating cycle of actions being interiorized into processes, which are encapsulated into objects, which together form schemas, is what gives APOS theory its name.

One of the book's strengths is its very clear description of APOS's research heuristic: the methods and approaches that researchers adopt within the programme. Fundamental to this are so-called 'genetic decompositions', hypothetical models that describe the mental actions, processes and objects that a student must develop, and the sequence in which they must develop them, to learn a given mathematical concept. APOS progresses by developing new genetic decompositions, testing them against empirical data pertaining to students' learning, and using them to design pedagogical materials. The book gives a number of detailed examples of genetic decompositions, and discusses the resulting pedagogical approaches.

I found the book's discussion of APOS's hard core and heuristic to be extremely clear and helpful. But there were two omissions which I felt could have strengthened it. First, APOS theory is a development of Piaget's notion of reflective abstraction, and this is reflected throughout the book by many favourable references to Piaget's research. But, although Piaget is a hugely influential scholar, his work has been heavily criticised. For instance, critics say that he was concerned only with description rather than explanation (e.g., Brainerd 1978), that many of the descriptions he gave are simply false (e.g., Fischer 1978; Johnson-Laird and Wason 1970), and that he dramatically underestimated the role of social factors in intellectual development (e.g., Broughton 1981). Given these complaints, many of which would seem to impact upon APOS theorists' use of his work, it seemed strange to find here only uncritical appeals to Piaget's thinking. Second, APOS theory is one of several process-to-object theories that have surfaced in the mathematics education literature. Closely related ideas are Sfard's (1991) notion of reification, and Gray and Tall's (1994) procept theory. Although the book does mention these rival theories, the discussion is dismissively limited to a single paragraph, which seemed like a missed opportunity.

I found the book's discussion of APOS's protective belt to be more problematic. As noted above, Lakatos (1976) suggested that the health of a research programme can be determined by how it deals with anomalies. If rescue hypotheses regularly need to be added to its protective belt, it is degenerating; if anomalies are incorporated so that novel predictions and results are generated, it is progressing. Few anomalies are discussed in this book, but it seems to me that there are many results from the mathematics education and mathematical cognition literatures which pose a challenge APOS's hard core. I give one example here.

Is it always the case that learners come to understand processes by interiorizing actions? APOS theory says yes, or at least that the "full development of a mathematical concept necessitates" this (p. 176). But there is a body of research which challenges this assertion, at least in the context of basic arithmetic (Gilmore and Bryant 2006, 2008; Gilmore and Papadatou-Pastou 2009; Sherman and Bisanz 2007). As noted above, if children come to understand the notion of addition as an object by first interiorizing actions and then encapsulating processes, we might expect that they would first gain increasing competence at completing addition problems (action), 
and then start to reverse these actions, and to reflect on them without needing to carry them out (process), before eventually being able to perform new actions on the process (object).

This account makes clear empirical predictions in the context of arithmetic inversion. Take the missing value problem $14+12-\square=17$. A child who is a skilled performer of the addition and subtraction actions would have few difficulties in solving the problem, whereas a child who was not yet sufficiently skilled with these actions would struggle. However, problems such as $13+\square-9=13$ or $15+13-\square=15$ have two possible solution strategies. They can be solved by the fluent application of actions in much the same way as the first problem, or they can be solved by inverting the appropriate addition or subtraction (i.e. by reversing the addition/subtraction, which according to APOS theory requires interiorization to have taken place). If the action-before-process account were correct, we would expect to see three types of children: those who are unable to do well on either problem type (who have yet to develop fluent actions), those who can solve calculation problems and inversion problems via a calculation strategy (who have fluent actions, but who have yet to interiorize these into processes), and those who can solve calculation problems with a calculation strategy and inversion problems with an inversion strategy (who have successfully interiorized their actions and so are able to think about addition/subtraction as processes and reverse them). We certainly would not expect to find students who are able to solve the inversion problems but not the calculation problems: such students would have interiorized an action without being able to perform it.

However, across a number of published studies, this latter group has regularly been identified. In their meta-analysis of 14 papers, Gilmore and Papadatou-Pastou (2009) found that roughly a third of children (220 out of the combined sample of 745) fell into this category. That is to say that a third of children appear to have a process conception of addition/subtraction, at least in the sense that they can reverse additions and subtractions, without being able to reliably perform the addition and subtraction actions. To be clear, I do not wish to suggest that this empirical finding falsifies APOS theory, or that the theory should be abandoned. But I do wish to claim that the finding is a clear example of a Lakatosian anomaly which APOS's protective belt needs to accommodate with some kind of rescue hypothesis.

A number of other well-established empirical results about mathematical development also seem hard to account for within APOS theory. For instance, if numbers are encapsulated processes, why do humans (and indeed animals) show numerical distance effects during comparison tasks (e.g., Moyer and Landauer 1967) or spatial/numerical associations during parity judgement tasks (e.g., Dehaene et al. 1993)? How does APOS theory account for developmental dyscalculia (e.g. Butterworth 2004)? Why do students often use linear reasoning in situations where it is inappropriate (e.g., Van Dooren et al. 2008)? All of these are well-established phenomena that a comprehensive theory of mathematical concept development ought to account for, at least using rescue hypotheses in its protective belt, if not using its hard core. The fact that the book does not address issues like this makes it difficult to judge the APOS research programme according to the principles set out by Lakatos (1976). 
In sum, for a reader interested in understanding APOS theory, this is an excellent book. It lays out APOS's theoretical assumptions and standard research methods with clarity and precision, and it gives helpful examples of research conducted within the programme. In Lakatos's (1976) sense, it gives a clear explication of APOS's hard core and heuristic. But I think the book will be much less successful at persuading non-APOS theorists to join the research programme. The book doesn't address fairly obvious Lakatosian anomalies, and one is left with the feeling that if APOS theorists were more engaged with the wider literature, the field would have a better sense of whether APOS is progressing or degenerating, and therefore whether or not it deserves wider attention.

\section{References}

Brainerd, C. (1978). The stage question in cognitive-developmental theory. Behavioural and Brain Sciences, 2, 173-213.

Broughton, J. (1981). Piaget's structural developmental psychology: 4. Knowledge without a self and without history. Human Development, 24, 320-346.

Butterworth, B. (2004). Developmental dyscalculia. In J. D. Campbell (Ed.), Handbook of mathematical cognition (pp. 455-467). New York: Psychology Press.

Dehaene, S., Bossini, S., \& Giraux, P. (1993). The mental representation of parity and number magnitude. Journal of Experimental Psychology: General, 122, 371-396.

Dubinsky, E. (1991). Reflective abstraction in advanced mathematical thinking. In D. O. Tall (Ed.), Advanced mathematical thinking (pp. 95-126). Dordrecht: Kluwer.

Fischer, K. (1978). Structural explanation of developmental change. Behavioural and Brain Sciences, 2, 186-187.

Gilmore, C., \& Bryant, P. (2008). Can children construct inverse relations in arithmetic? Evidence for individual differences in the development of conceptual understanding and computational skill. British Journal of Developmental Psychology, 26, 301-316.

Gilmore, C., \& Papadatou-Pastou, M. (2009). Patterns of individual differences in conceptual understanding and arithmetical skill: a meta-analysis. Mathematical Thinking and Learning, 11, 2540.

Gilmore, C. K., \& Bryant, P. (2006). Individual differences in children's understanding of inversion and arithmetical skill. British Journal of Educational Psychology, 76, 309-331.

Gray, E. M., \& Tall, D. O. (1994). Duality, ambiguity and flexibility: a proceptual view of simple arithmetic. Journal for Research in Mathematics Education, 25, 115-141.

Johnson-Laird, P. N., \& Wason, P. C. (1970). A theoretical analysis of insight into a reasoning task. Cognitive Psychology, 1, 134-148.

Kuhn, T. S. (1962). The structure of scientific revolutions. Chicago: University of Chicago Press.

Lakatos, I. (1976). The methodology of scientific research programmes. Cambridge: Cambridge University Press.

Moyer, R. S., \& Landauer, T. K. (1967). Time required for judgements of numerical inequality. Nature, $215,1519-1520$.

Selden, A. (2012). A home for RUME: the story of the formation of the mathematical association of America's special interest group of research in mathematics education. Technical Report 2012-6, Tennessee Technological University, Cookeville.

Sfard, A. (1991). On the dual nature of mathematical conceptions: reflections on processes and objects as different sides of the same coin. Educational Studies in Mathematics, 22, 1-36.

Sherman, J., \& Bisanz, J. (2007). Evidence for use of mathematical inversion by three-year-old children. Journal of Cognition and Development, 8, 333-344.

Tall, D.O. (1991). Advanced mathematical thinking. Dordrecht: Kluwer.

Van Dooren, W., De Bock, D., Janssens, D., \& Verschaffel, L. (2008). The linear imperative: An inventory and conceptual analysis of students' overuse of linearity. Journal for Research in Mathematics Education, 39, 311-342. 